\title{
Australian guideline for treatment of problem gambling: an abridged outline
}

Shane A Thomas BA, DipPubPoli, PhD Directo

Stephanie S Merkouris BPsych, GradDipPsych Research Assistant

Harriet L

Radermache BSc, DPsych, Research Fellow

Nicki A Dowling BSc, MPsych(Clinical), PhD Senior Research Fellow

Marie L Misso PhD, BSc(Hons) Head of Evidence Synthesis Program

Christopher J Anderson BScEd, BTheol(Hons) Research Fellow

Alun C Jackson BA, MSc, PhD, Director ${ }^{2}$

1 Problem Gambling Research and Treatment Centre, Monash University, Melbourne, VIC

2 Problem Gamblin Research and Treatment Centre, University of Melbourne, Melbourne, VIC

3 Jean Hailes Foundation for Women's Health, Schoo of Public Health and Preventive Medicine Monash University, Melbourne, VIC.

shane.thomas@ monash.edu

doi: 10.5694/mjall.11088

A longer version of this article was published online $22 / 11 / 11$

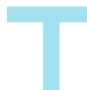

he Problem Gambling Research and Treatment Centre (PGRTC) has developed the first evidence-based guideline to address problem gambling in Australia - Guideline for screening, assessment and treatment in problem gambling. The entire guideline and related appendices have been approved by the Chief Executive Officer of the National Health and Medical Research Council (NHMRC) under s.14A of the National Health and Medical Research Council Act 1992. In approving the guideline, the NHMRC considered that it meets the NHMRC's standard for clinical practice guidelines. The guideline will be available on the PGRTC websites (http://www.med.monash.edu.au/sphc/ pgrtc and http://www.education.unimelb.edu.au/problemgambling). The full guideline addresses screening, assessment and treatment issues relating to problem gambling; in this abridged outline, we focus solely on treatment interventions for problem gamblers.

\section{Definition and prevalence of problem gambling}

A range of terms have been used to describe problematic gambling, including pathological, disordered, compulsive and problem gambling. In Australia, "problem gambling" is the most commonly used term and describes the situation in which a person has "difficulties in limiting money and/or time spent on gambling which leads to adverse consequences for the gambler, others, or for the community". ${ }^{1}$ The Productivity Commission found that the prevalence of problem gambling was approximately $2.1 \%$ of the adult Australian population. ${ }^{2}$ Recent statewide gambling surveys suggest that between $1.4 \%$ and $3.1 \%$ of adults report problem or moderate-risk gambling, based on the Problem Gambling Severity Index (PGSI) of the Canadian Problem Gambling Index. ${ }^{3-9}$ Some variation in rates between states may be the result of variable administration of the PGSI. ${ }^{10}$

\section{Guideline development process}

A comprehensive and systematic process was used to develop the guideline (Box 1), as outlined in the NHMRC standards and procedures for externally developed guidelines. ${ }^{11}$ Due to the impact of internal validity on studies of interventions for problem gambling, articles were only included if they were classified as Level I or Level II evidence.

\section{Treatment recommendations}

A total of 34 randomised controlled trials, reported in 37 articles, met the inclusion criteria for the clinical questions and formed the basis of seven evidence-based recommendations for treatment (Box 2). Each recommendation was accompanied by a grade that reflected the volume, consistency, clinical impact, generalisability and applicability of the evidence (Box 3), and practice points to provide practical advice and information.

\section{Considerations for practice}

The recommendations are expected to result in a consolidation of current practices in the treatment of problem gambling in Australia. CBT is already widely used as the therapy of choice for problem gambling and is a standard component in clinical training curricula for psychology and psychiatry. In comparison, motivational interviewing and motivational enhancement therapy are not commonly included in standard training programs, and practitioners who wish to deliver these interventions for problem gambling should undertake training. Furthermore, as outlined in the practice points, appropriately qualified and trained practitioners are advised to consider client preferences, availability of services, and manualised delivery of any chosen intervention. These considerations are especially pertinent given that practitioner-delivered psychological interventions largely underpinned the recommendations and, importantly, were found to be more effective than self-help interventions.

Two of the seven recommendations related to pharmacological interventions, and only one of these provided support for the use of a medication (naltrexone) to reduce gambling severity. Despite this recommendation, pharmacological interventions for the treatment of problem gambling should be applied with caution and with careful consideration of each patient's needs. Specific details in the product information for each drug regarding dosage, adverse effects, method and route of administration, and contraindications should be studied and followed carefully.

1 Guideline development process

The Guideline Development Group and the multidisciplinary Expert Advisory Panel devised 22 clinical questions regarding treatment

- A broad-ranging and comprehensive systematic search was done to identify all available literature

All identified articles were scanned to determine if they met the predefined inclusion or exclusion criteria

Data were extracted and critical appraisal of each included study was undertaken

Sufficient evidence was found to make evidence-based recommendations for six clinical questions 


\section{Evidence-based treatment recommendations and practice points}

\section{Recommendation (evidence grade)}

Individual or group cognitive behaviour therapy (CBT) should be used to reduce gambling behaviour, gambling severity and psychological distress in people with gambling problems (Grade B) Motivational interviewing and motivational enhancement therapy should be used to reduce gambling behaviour and gambling severity in people with gambling problems (Grade B)

Practitioner-delivered psychological interventions should be used to reduce gambling severity and gambling behaviour in people with gambling problems (Grade B)

Practitioner-delivered psychological interventions should be used over self-help psychological interventions to reduce gambling severity and gambling behaviour in people with gambling problems (Grade B)

Group psychological interventions could be used to reduce gambling behaviour and gambling severity in people with gambling problems (Grade C)

Antidepressant medications should not be used to reduce gambling severity in people with gambling problems alone (Grade B)

Naltrexone could be used to reduce gambling severity in people with gambling problems (Grade C)

As yet, no drugs have been approved by the Australian Therapeutic Goods Administration in the form of a registered indication or approved use for treatment of problem gambling. However, this does not preclude the use of drugs for non-registered indications or off-label prescribing. Registered indications ensure that the appropriate research and approval processes have been followed to ensure effectiveness and safety of the drug.

While the clinical questions and inclusion/exclusion criteria developed for the guideline ensured that all relevant evidence was retrieved with respect to different subgroups (based on co-occurring psychiatric symptoms, sex, age, and gambling type), the paucity of available evidence means that the final recommendations must be applied with caution with respect to specific groups. However, this limitation in the evidence base has been acknowledged and accounted for in the formulation and grading of the recommendations.

\section{Considerations for research}

Given the current immaturity of the research literature in the field of problem gambling, relevant and high-quality evidence could be found to address only six of the 22 clinical questions regarding treatment, leading to the formulation of only seven evidence-based recommendations. This outcome was not unexpected. Rather, it was intended that by conducting this review, formal identification of the gaps in knowledge would assist the strategic advancement of the field through targeted research and development, as well as in guiding practitioners regarding the evidence available to inform their practice. The Guideline Development Group made several recommendations for further research that can be found in the full guideline.

\section{Practice points}

Where CBT is to be prescribed, the following could be considered

Appropriate qualifications and training of practitioners

Manualised delivery of the intervention

Where motivational interviewing and motivational enhancement therapy are to be prescribed, the following could be considered:

- Appropriate qualifications and training of practitioners

- Manualised delivery of motivational enhancement therapy

Where practitioner-delivered psychological interventions are to be prescribed, the following could be considered:

- Client preferences Appropriate qualifications and training of practitioners Availability of services Manualised delivery of the intervention

Where practitioner-delivered psychological interventions are to be prescribed, the following could be considered:

- Client preferences - Appropriate qualifications and training of practitioners

- Availability of services Manualised delivery of the intervention

Where group psychological interventions are to be prescribed, the following could be considered: Client preferences Appropriate qualifications and training of practitioners Availability of services Manualised delivery of the intervention

- Due to the nature of the samples studied, this recommendation is applicable to those with gambling problems only, and not those who may have comorbidities such as depression and anxiety

This recommendation is predominantly based on evidence evaluating the effectiveness of selective serotonin reuptake inhibitors

Where naltrexone is to be prescribed, the following could be considered:

Problem gambling is not (at the time of reporting) a registered indication for naltrexone, so a Pharmaceutical Benefits Scheme subsidy would not apply for this indication

Appropriate skills and training of the prescribing practitioner

Recommended contraindications

\section{Grades of recommendations}

\section{Grade Description}

A Body of evidence can be trusted to guide practice

B Body of evidence can be trusted to guide practice in most situations

C Body of evidence provides some support for recommendation but care should be taken in its application

D Body of evidence is weak and recommendation must be applied with caution

Acknowledgements: We acknowledge the important contribution of the Expert Advisory Panel as well as the technical and operational support provided by Sean Cowlishaw, Felicity Lorains, Anna Chapman and Sylvia Niele.

Competing interests: No relevant disclosures.

1 Neal P, Delfabbro P, O’Neil M. Problem gambling and harm: towards a national definition. Melbourne: Gambling Research Australia, 2005.

2 Productivity Commission. Australia's gambling industries. Report No. 10. Canberra: AusInfo, 1999.

3 Davidson T, Rodgers B. 2009 Survey of the nature and extent of gambling, and problem gambling, in the Australian Capital Territory. Canberra: ACT Gambling and Racing Commission, 2010.

4 Hare S. A study of gambling in Victoria - problem gambling from a public health perspective. Melbourne: Department of Justice, 2009.

5 ACNielsen. Prevalence of gambling and problem gambling in New South Wales - a community survey 2006: final report. Sydney: NSW Office of Liquor, Gaming and Racing, 2007.

6 Queensland Department of Employment, Economic Development and Innovation. Queensland Household Gambling Survey 2008-09. Brisbane: Queensland Government, 2010.

7 O'Neil M, Chandler N. Kosturjak A, et al. Social and economic impact study into gambling in Tasmania. Adelaide: South Australian Centre for Economic Studies, 2008.

8 South Australian Department for Families and Communities. Gambling prevalence in South Australia: October to December 2005. Adelaide: South Australian Department for Families and Communities, 2006.

9 Young M, Abu-Duhou, I Barnes T, et al. Northern Territory Gambling Prevalence Survey 2005. Darwin: Charles Darwin University, 2006.

10 Jackson AC, Wynne H, Dowling NA, et al. Using the CPGI to determine problem gambling prevalence in Australia: measurement issues. Int $\mathrm{J}$ Ment Health Addict 2010; 8: 570-582

11 National Health and Medical Research Council. NHMRC standards and procedures for externally developed guidelines. Canberra: NHMRC, 2007. 